\title{
Analysis of Spectrum Utilization in Suburb Environment - Evaluation of Potentials for Cognitive Radio
}

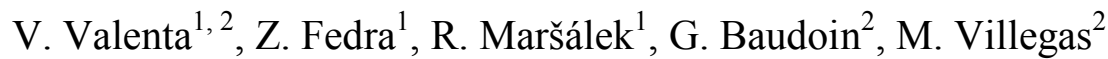 \\ ${ }^{1}$ Dept. of Radio Electronics, Brno University of Technology, Brno, 61200 Czech Republic \\ ${ }^{2}$ Université Paris-Est, ESYCOM, ESIEE, Cité Descartes, 93162 Noisy-le-Grand, France \\ \{valentav, baudoing,villegam\}@esiee.fr, \{fedraz, marsaler\}@feec.vutbr.cz
}

\begin{abstract}
This paper deals with spectrum utilization measurements in the frequency band from $100 \mathrm{MHz}$ up to $3 \mathrm{GHz}$. The measurement method is based on the energy detection principle using wideband logperiodic antenna. Our results show that the radio spectrum is not utilized in an optimal manner and that there do exist sporadically utilized licensed frequency bands that could be possibly used by cognitive radios in an opportunistic way. Cognitive radio concept for better spectrum utilization is introduced here along with an overall approach regarding spectrum utilization in the next generation wireless networks.
\end{abstract}

\section{Introduction}

The electromagnetic spectrum is a scarce natural resource and as any other finite resource, it is limited by its usability. Therefore, the electromagnetic spectrum has to be regulated by relevant governmental authorities (ITU, ČTÚ, FCC etc). Actual wireless communication systems are mainly based on fixed resource allocation. The radio spectrum is assigned or sold to users on long term basis, covering large regions like whole countries. However, as many measurement studies reveal [1], [2], this approach to the frequency allocation leads into vast underutilization of frequency spectrum due to very sporadic usage within various geographical regions as well as over a given period of time. These results lead us to reconsider the allocation protocol of the frequency spectrum and moreover, it opens space for novel and more efficient wireless communication systems that will be based on dynamic spectrum allocation (DSA). This basic idea is the keystone of the Cognitive Radio (CR) concept, a technology that envisages a flexible access to unused or underutilized frequency spectrum from primary licensed networks and consequently solves the spectrum utilization problem. The fundamental concept of the CR has been firstly proposed by J. Mitola III [3] and can be described as a software defined radio (SDR) based system that senses and is aware of its operational environment and can dynamically and autonomously adjust its radio operating parameters [4]. The key attributes of the CR are collaborative spectrum sensing (awareness of its surroundings) and adaptation ability of transmission features (TX frequency, technique of transmission, direction of transmission by means of beamforming etc).

Spectrum agility and the overall adaptation process of the CR are shown in Fig. 1. Firstly, spectrum sensing and signal waveform determination functions are employed in order to find proper transmission resources, so called "spectrum holes". This function can be based on the real-time spectrum scanning and moreover, on the knowledge of the local environment (e.g. a regional frequency allocation database and utilization statistics downloaded via cognitive pilot channel [5]). Next, the best course of action upon spectrum findings is taken. This action includes a new allocation of resources and process initiation. Finally, the reconfigurable SDR based transceiver is adjusted accordingly. This whole process is dynamic and has to be updated regularly, according to the space-time spectrum availability and the actual transmission request (data rate, BER, latency, etc).

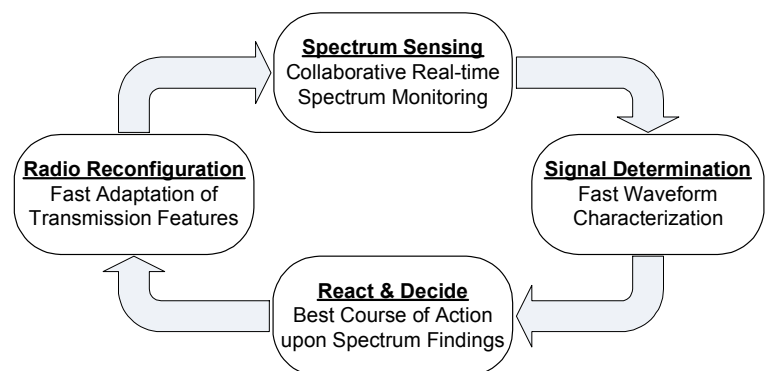

Figure 1. Spectrum agility process of the CR: from spectrum sensing and intelligent decision to reconfiguration [8]. 
It is obvious that the correct determination of spectrum holes and the ability to estimate the utilization is a very critical issue for DSA algorithms and hence, understanding the spectrum utilization becomes very important. Long term statistical measurements of spectrum utilization could be possibly used and regularly updated by CRs and measurement stations in particular geographical regions in order to facilitate finding of unused and underutilized spectrum gaps. In this context, the main objective of this paper is to present the behavior of the radio spectrum utilization in the typical suburban environment and to point out on certain poorly utilized bands that could be dynamically accessed by future opportunistic wireless devices. The measurement method, measurement equipment, data processing and major conclusions of the spectrum utilization analysis will be presented in following sections respectively along with perspectives and recommendations for the future research.

\section{Measurement method}

Spectrum utilization measurement has been carried out on the roof of the Department of Radioelectronics building, which is located in the northern suburb of the town of Brno in the Czech Republic. A wideband measurement antenna has been pointed towards the town in three directions day by day in order to cover the whole urban area. Vertical and horizontal polarization of the logarithmically periodic antenna has been considered. This measurement is different to those implemented in other studies (e.g. [1], [2]) since we focus on the urban spectrum utilization seen from the suburb location. As it will be shown later on, certain frequency bands in this area exhibit much lower utilization than expected and therefore, the frequency reuse by the $\mathrm{CR}$ can be even more extended.
Measurements have been carried out during six subsequent days - three days using vertical polarization and three days with horizontal polarization. The overall result of this measurement is depicted in Fig. 2. Indepth utilization analysis of selected bands will be presented in Section 2.

\section{A. Measurement equipment}

The signal captured by the wideband antenna has been analyzed by the spectrum analyzer Rohde\&Schwarz FSP and then loaded via GPIB/USB to PC using Matlab Instrument Control Toolbox. Matlab has been used for signal analysis of stored data. Radiation diagram of the log-periodic antenna at 1.5 $\mathrm{GHz}$ is depicted in Fig. 3. It can be seen that the directivity is relatively low and therefore, only three directions can be analyzed in order to cover the main part of the town. Correction factors due to the antenna gain, cable loss and connections have been considered as well.

\section{B. Data processing}

The whole frequency band of $2.9 \mathrm{GHz}$ (from 100 $\mathrm{MHz}$ to $3 \mathrm{GHz}$ ) has been divided into $14520 \mathrm{MHz}$ sub-bands. In order to achieve high resolution, each sub-band has been analyzed using resolution bandwidth of $3 \mathrm{kHz} .125$ samples have been stored for every single $20 \mathrm{MHz}$ sub-band, which in turn makes 18,125 samples per the whole analyzed bandwidth. 125 samples per sub-band corresponds to one sample every $160 \mathrm{kHz}$. Sweep time of 4 seconds has been set for every $20 \mathrm{MHz}$ sub-band and hence, the total scanning time of the $2.9 \mathrm{GHz}$ band results in 580 seconds. This measurement has been performed repeatedly during 24 hours for every one of three antenna directions and both antenna polarization.

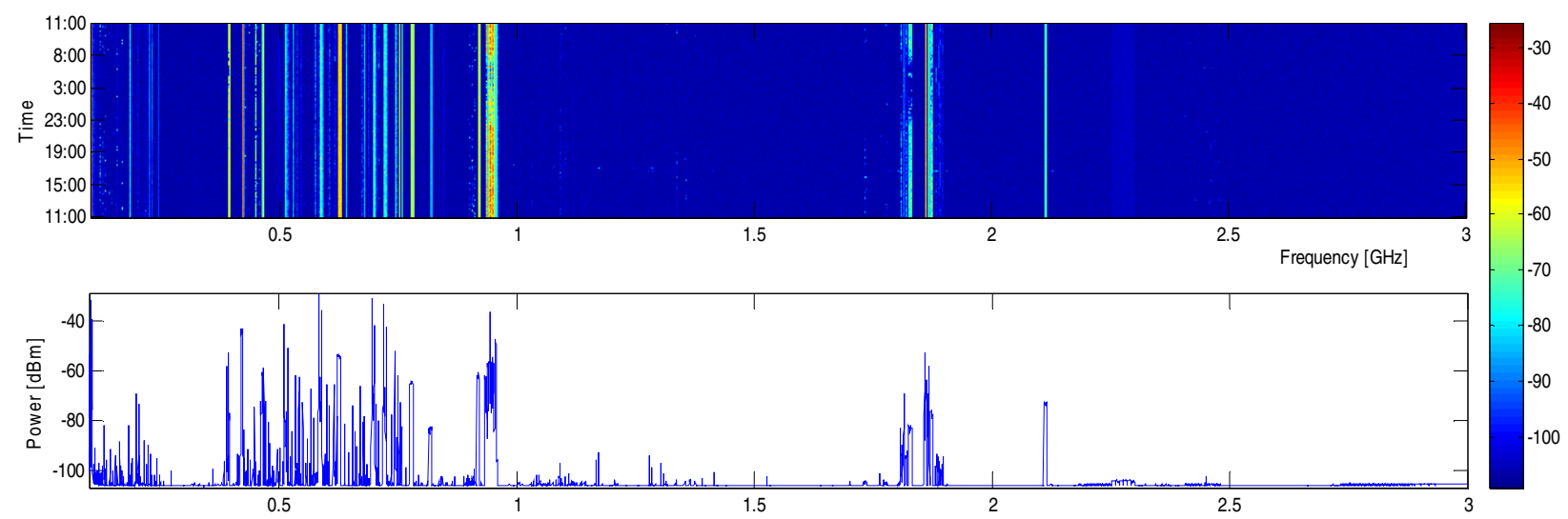

Figure 2. Overall result of the temporal and the average radio spectrum occupancy (colorbar in $\mathrm{dBm})$. Different activity in time is noticeable. 


\section{Spectrum utilization analysis}

Figure 2 displays only rough information of the whole band of interest. In order to get more characteristic information about the spectrum utilization, we will zoom on selected licensed frequency bands and analyze them in a detail. Following frequency bands have been chosen: 380 385/390-395 MHz (TETRA), 410.2-412.8/420.2$422.8 \mathrm{MHz}$ (CDMA), 451.5-455.54/461.5-465.54 $\mathrm{MHz}$ (the first CDMA2000 in $450 \mathrm{MHz}$ ), 470-862 $\mathrm{MHz}$ (TV), 888-915/933-960 MHz (E-GSM 900), 1710-1785/1805-1880 MHz (GSM 1800), 19201980/2110-2170 MHz (UMTS TDD), 2400-2483.5 $\mathrm{MHz}$ (ISM) and other bands.

The utilization of selected frequency bands has been defined by the duty cycle parameter, which specifies the fraction of time the band is used. The duty cycle in selected frequency bands has been calculated as a ratio of the number of samples $N_{(P>t h r e s h o l d)}$ with power level superior to the power threshold and the total number of samples $N_{\text {Total }}$.

$$
\text { Duty Cycle }=\frac{N_{(\text {P }>\text { threshold })}}{N_{\text {Total }}}
$$

Determination of the power threshold is a critical issue. An excessive threshold may lead to overlooking potential activity within the band, but on the other hand, too low values may result in false alarm due to the background noise. In order to overcome this problem, various thresholds for different frequency bands have been defined, depending on the level of the background noise. The power threshold that determines the occupancy of a frequency sample has been set in most cases to $7 \mathrm{~dB}$ above the maximum average value of the background noise measured around the actual band. $7 \mathrm{~dB}$ has been chosen as the optimal value based on long term observation of the background noise.

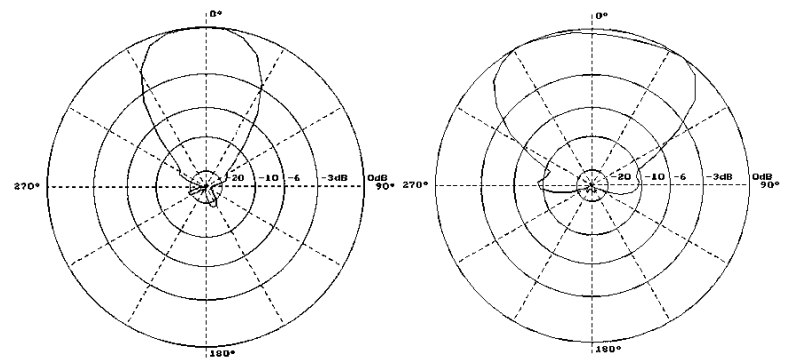

Figure 3. Radiation diagram of the wideband log-periodic antenna at $1.5 \mathrm{GHz}$ in the vertical (left) and horizontal plane (right).
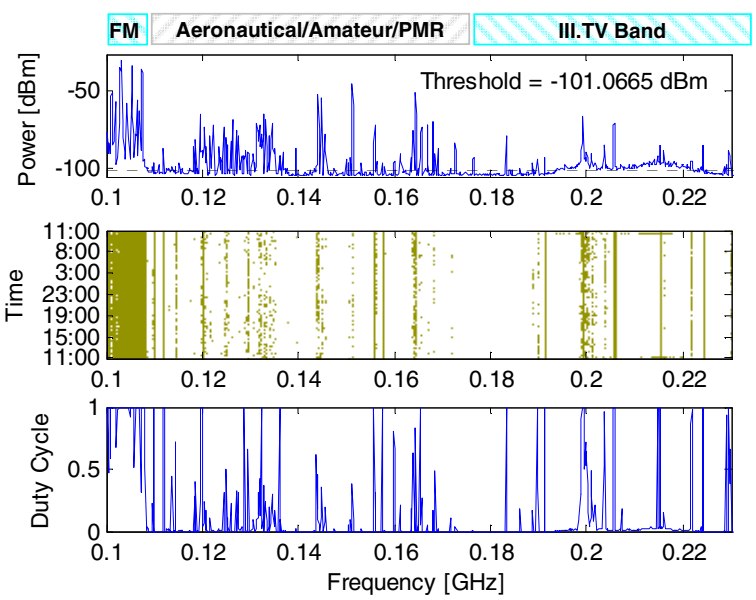

Figure 4. FM radio, aeronautical, amateur and PMR applications and analogue TV channels in the III. TV band.

However, in some cases, the threshold has been deliberately decreased in order to pick out very weak signals otherwise considered as a background noise (ISM and MMDS in the $2 \mathrm{GHz}$ band, see Fig. 10).

Following figures (Fig. 4-10) provide a detailed analysis of selected frequency bands. Each triplet of charts presents the maximum received power, frequencies considered as occupied and the corresponding duty cycle respectively. Maximum received power representation was preferred to the average power plot due to the capability to display more representative frequency profiles of considered signals. Therefore, the difference between the power threshold and the background noise depicted in figures does not correspond to above mentioned $7 \mathrm{~dB}$.

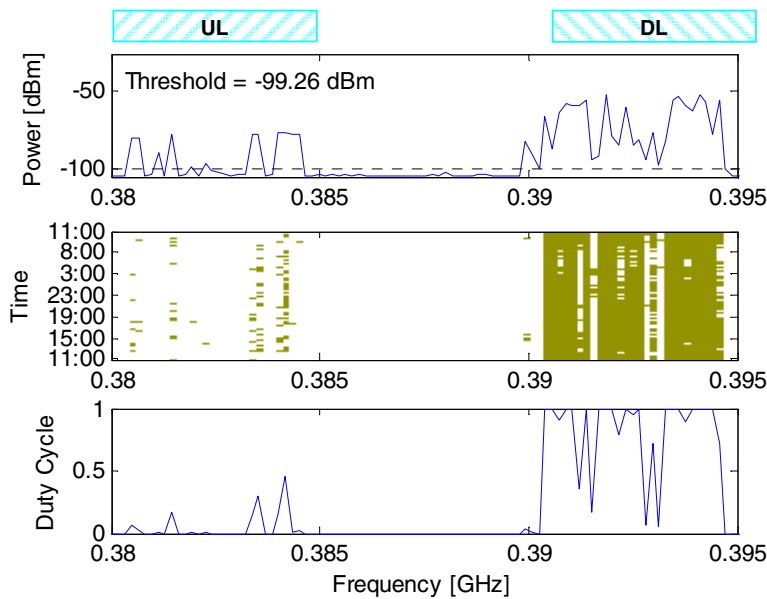

Figure 5. PMR communication system TETRA with duplex links in the band of 380-385/390$395 \mathrm{MHz}$. 


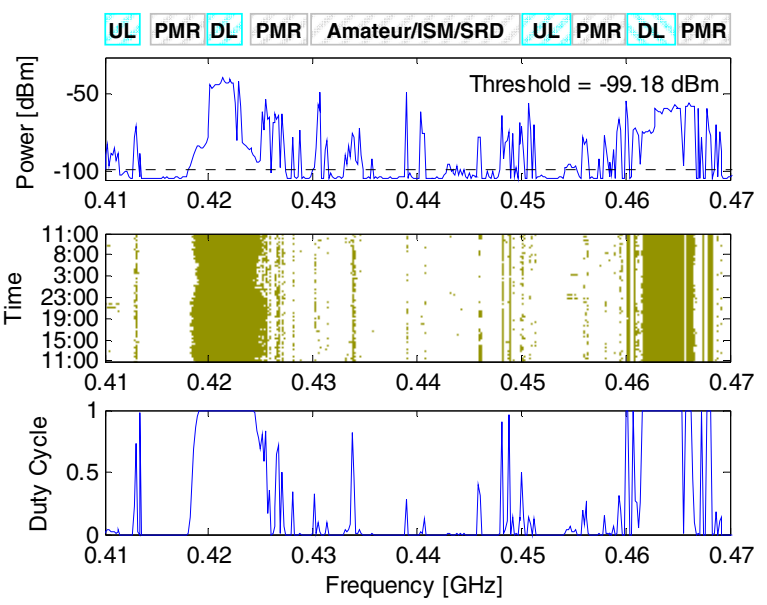

Figure 6. Two different CDMA2000 systems in duplex bands of 410.2-412.8/420.2-422.8 and 451.5-455.54/461.5-465.54 MHz.

Frequency utilization within the band of 100-230 $\mathrm{MHz}$ is $12.7 \%$. This band is mainly allocated to the broadcasting (FM radio up to $108 \mathrm{MHz}$, III. TV band in 174-230 MHz), aeronautical applications and the PMR (Professional Mobile Radio) service [9]. Nearly continuous utilization exhibits the FM radio band. On the other hand, the III. TV band performance reveals a large underutilization.

Figures 6,8 and 10 show performance of CDMA based systems, namely CDMA2000 EV-DO in 400 $\mathrm{MHz}$ band and two UMTS TDD systems in $900 \mathrm{MHz}$ and $2.1 \mathrm{GHz}$ band. Due to the nature of the wideband CDMA, only downlink channels (DL) have been detected. These DL channels have been fully utilized during six subsequent days, which in turn leads to
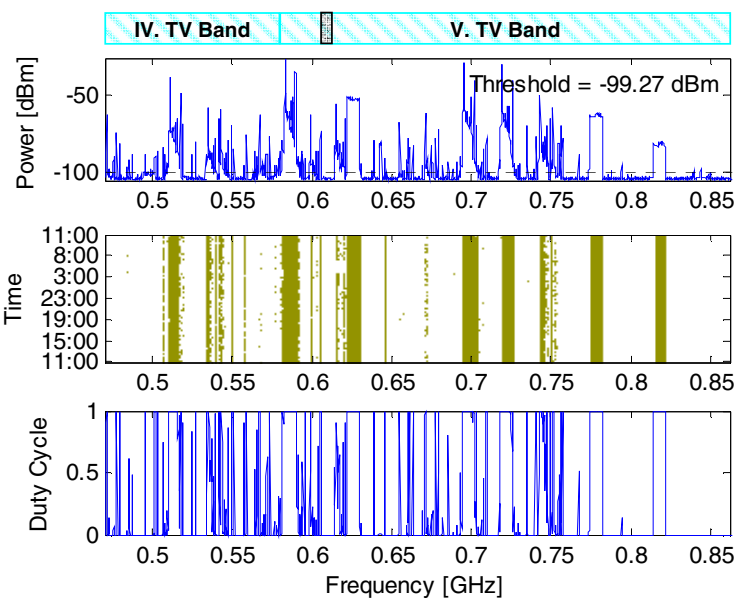

Figure 7. IV. and V. TV band, analogue and DVB-T in the band of 470-862 MHz. Notice the different power spectrum of analogue and OFDM based DVB-T channels.
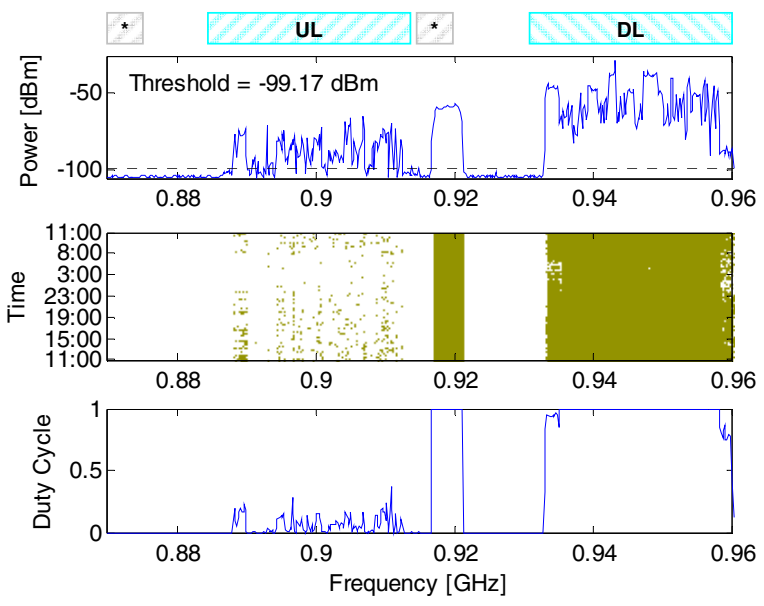

Figure 8. E-GSM in band of 888-915/933-960 MHz and UMTS TDD in band of 872-876/917$921 \mathrm{MHz}$ (asterisk field ${ }^{\star}$ ).

approximately $50 \%$ utilization. Utilization of the UMTS TDD system in the $2.1 \mathrm{GHz}$ band is much lower (only $3.8 \%$ ). This is due to the fact, that only one $5 \mathrm{MHz}$ channel out of $60 \mathrm{MHz}$ DL allocation has been active. Uplink (UL) activity has not been detected, because the energy of the CDMA signal is spread over a wide band in order to transmit lower power. Therefore, such noise-like signals are hard to detect using energy detection principle. Detection of these signals is generally based on feature detection due to very distinct spectral correlation properties of wideband signals [7].

Utilization of TV bands presents a significant frequency reuse opportunity. Moreover, this radio band will offer even more possibilities after complete transition to the more efficient digital TV, which will consequently free up new spectrum for future wireless applications. It has to be noted that this part of the radio spectrum is particularly precious, because the propagation of such electromagnetic waves exhibits very good properties in terms of penetration and coverage range. Therefore, special attention should be paid to the radio resource management in this particular frequency band. As seen in Fig. 7, many TV channels appear to be unused or are very weak. This is due to the fact that the TV channel allocation depends on the geographical location. Ten out of 49 allocated channels were considered as occupied, which in turn results in $20.4 \%$ utilization of the whole TV band. This result does not consider TV transmitters located on the other side of interest and, therefore, the actual utilization might be considerably higher. In order to get more appropriate utilization results of TV bands, omnidirectional measurements and analysis has been carried out. However, our results of the omni directional 

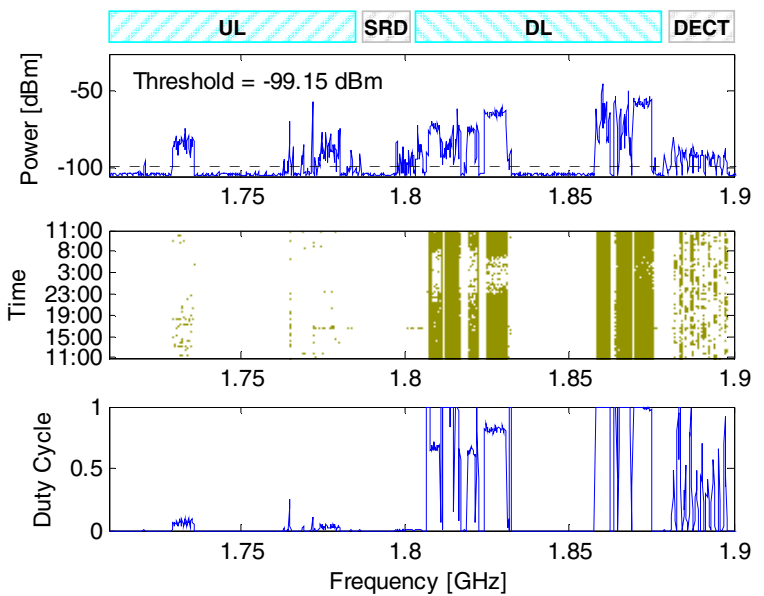

Figure 9. GSM 1800 in band of 1710 1785/1805-1880 MHz and the cordless system DECT in band of 1880-1900 MHz.

measurement did not reveal any higher utilization within this band. The only difference was found to be the received power level of already detected TV channels.

Utilization of TETRA and GSM systems very much depends on the period of time (Fig. 5, Fig. 8-9). A significant decrease of activity was measured during the night time, from $11 \mathrm{PM}$ to $7 \mathrm{AM}$. It has been observed that downlink channels exhibit much higher utilization than uplink channels. This is due to the fact that the transmitting power of base stations is considerably higher than the power transmitted by individual mobile stations and moreover, the power attenuation due to the built-up area is much lower in case of base stations, since they are situated on top of buildings. Performance of the cordless communication
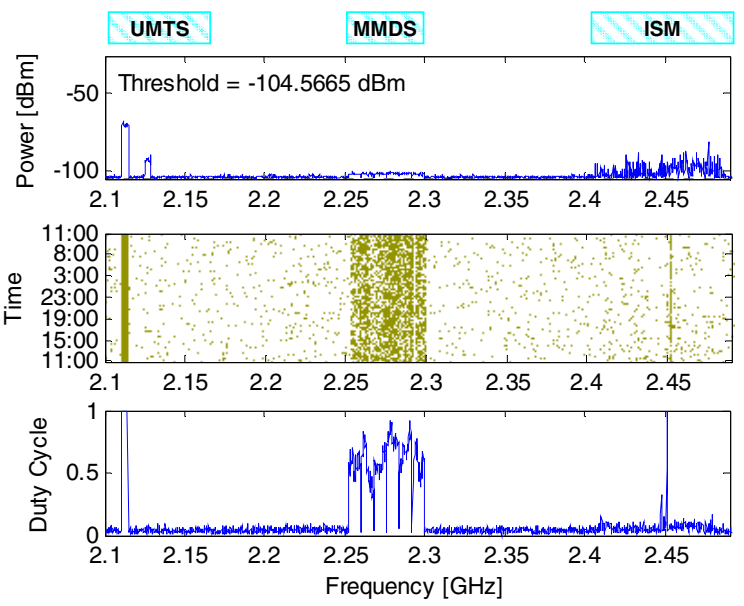

Figure 10. UMTS DL channel in band of 2.112.17 GHz, MMDS system in 2.25-2.3 GHz and ISM band 2.4-2.483 GHz. system DECT is depicted in Fig. 9. Each of ten carriers in the band of 1880-1900 MHz exhibits almost equally distributed traffic, which results in $22.1 \%$ utilization. Apart from this, Figure 9 demonstrates very low utilization in the band of $1795-1805 \mathrm{MHz}$, which is allocated to Short Range Devices (SRD).

Figure 10 depicts utilization performance in the band of 2-2.48 GHz. This radio band covers UMTS DL TDD channel, Microwave Multipoint Distribution Service (MMDS) and the ISM band [9]. Power threshold value has been purposely shifted down to $-104.6 \mathrm{~dB}$ due to very low power level detected at the antenna. By doing so, we can pick up very weak radio signals, but on the other hand, this measure results in adding extra noise, as seen in Fig. 10. MMDS exhibits almost continuous traffic and utilization of $58 \%$. Very low utilization performance has been measured in the $2.4 \mathrm{GHz}$ ISM band (less than $1 \%$ when using a threshold of $-102 \mathrm{dBm}$ ). One explanation is that most of wireless systems working in this band use highly directive antennas pointing downwards to the ground in order to cover small access spots. Moreover, electromagnetic propagation properties in the $2.4 \mathrm{GHz}$ band in the urban area are very poor. It is obvious that this particular band would exhibit different performance in the downtown area and therefore, corresponding analyses need to be carried out in such a specific environment.

\section{Conclusion}

Utilization of the radio spectrum has been measured and behaviour of selected licensed bands has been analyzed in a detail (see Table 1 for summary of results). It has been shown that the total spectrum utilization in the radio band of $100 \mathrm{MHz}$ to $3 \mathrm{GHz}$ is lower than $6.96 \%$. These results demonstrate the fact

Table 1. Spectrum utilization summary of selected communication standards.

\begin{tabular}{|c|c|c|}
\hline System & Frequency [MHz] & Utilization [\%] \\
\hline TETRA & $380-385 / 390-395$ & 37.5 \\
\hline CDMA & $\begin{array}{c}410.2-412.8 / 420.2-422.8 \\
451.5-455.54 / 461.5-465.54\end{array}$ & $50.7 / 47.2$ \\
\hline TV & $470-862$ & 20.4 \\
\hline E-GSM & $888-915 / 933-960$ & 51.9 \\
\hline GSM 1800 & $1710-1785 / 1805-1880$ & 21.8 \\
\hline DECT & $1880-1900$ & 22.1 \\
\hline UMTS TDD & $1920-1980 / 2110-2170$ & 3.8 \\
\hline ISM & $2400-2483.5$ & 1.0 \\
\hline TOTAL & $100-3000$ & 6.96 \\
\hline
\end{tabular}


that there is a lot of underutilized space in the frequency spectrum, which could be used by cognitive radio technologies based on dynamic spectrum access. Our results reveal spectrum utilization in particular environment however, this work is still preliminary. It is obvious that further in-depth studies are necessary to get more appropriate data, e.g. spectrum utilization analyzed in an appropriate statistical manner over much longer period (months) and wide range of locations and various environments. Currently, we are analyzing measurement results from identical utilization measurements that have been carried out in Parisian region in France. Moreover, similar measurement will be carried our in rural areas in the Czech Republic. Based on such a long term analysis, we will be able to determine, which licensed bands are more or less suitable for dynamic spectrum access, and which long-standing licensed bands should be reallocated to new services. Long term analyses will moreover reveal actual trend of spectrum utilization within particular radio bands, correlation between different locations and will furthermore serve as input values for CR learning algorithms.

\section{Acknowledgement}

The research has received funding from the European Community's Seventh Framework Programme under grant agreement no. 230126 and partially by the Czech science foundation project 102/09/0776, 102/08/H027 and COST IC0803 RFCSET.

\section{References}

[1] D'Iitri, S., McHenry, M. Dynamic spectrum access moves to the forefront. Defence Electronics. April 2008, p. S3 - S6.

[2] Habibul, I. et al. Spectrum survey in Singapore: occupancy measurements and analyses. $3^{\text {rd }}$ Int. Conference on Cognitive Radio Wireless Networks and Communications. Singapore, 2008.

[3] Mitola, J. et al. Cognitive radios: making software radios more personal, IEEE Personal Communications. August 1999 vol. 6 , no. 4 , pp. 13-18.

[4] Jondral, F. K. From Maxwell's equations to cognitive radio. $3^{\text {rd }}$ Int. Conference on Cognitive Radio Wireless Networks and Communications. Singapore, 2008.

[5] Houze, P., Ben Jemaa, S., Cordier, P. Common pilot channel for network selection. VTC - Spring Conference. Melbourne, 2006.

[6] Valenta, V., Fedra, Z., Marsalek, R., Baudoin, G., Villegas, M. Towards cognitive radio networks: spectrum utilization measurements in suburb environment. To appear in Radio Wireless Symposium '09, January 2009.

[7] Gardner, W., Brown III, W., Chen, C.-K. Spectral correlation of modulated signals: Part II - digital modulation. IEEE Trans. Communications. June 1987, vol. COM-35, no. 6, pp. 595-601.

[8] Evans, J. B. Intelligence in th Network. Keynote speech, Available: http://www.crowncom.org.

[9] Radio spectrum utilization plan determined by the Czech Telecommunication Office. Available: http://www.ctu.eu. 